\title{
The Transmission of Chinese Medicine in Australia
}

\author{
Mary Garvey, University of Technology, Sydney
}

\section{Introduction}

Chinese medicine is a complex field with a very long history and a great many diverse currents. Today, mainland Chinese still use Chinese medicine (CM) for the treatment of a wide range of medical conditions, and China's medical students study CM alongside Western biomedicine because the nation's integrated healthcare system delivers both. Australians also use CM for all kinds of acute and chronic illnesses even though Australian CM practitioner training qualifies its graduates to practice Chinese acupuncture and herbal medicine only. A brief overview of CM in China and Australia below will highlight some of the factors that have influenced its evolution over the last century, its transmission to Australia, and the continued challenges to its transmission in Australia.

The transmission of CM within and outside of China has historically been possible largely due to the textual legacy that has recorded its conceptual and therapeutic developments. While there are a few earlier sources from the Warring States period (476-221 BCE), China's literary medical traditions really began in the Han dynasty (206 BCE-220 CE) with the compilation of its earliest and most famous medical classics: the Yellow Emperor's Internal Canon (黄帝内经 Huangdi Neijing c.100 BCE); the Canon of Difficult Issues (难经 Nanjing c.150 CE); and the Treatise on Cold Damage and Miscellaneous Disorders (伤寒杂病论 Shanghan Zabing Lun c.200 CE).

PORTAL Journal of Multidisciplinary International Studies, vol. 8, no. 2, July 2011. 
In Chinese libraries today there are over 12,000 pre-modern medical texts covering the period since the Han, but only a few have been translated into modern languages in a philologically serious way (Unschuld 1993: 24). Moreover, translations, technical language and terminology to date have been largely interpretive, idiosyncratic and difficult to cross-reference.

Because the practitioner's image of the medical body guides their approach to treatment, education and training have consequences for clinical practice and for the development of $\mathrm{CM}$ as a distinct discipline and healthcare profession. In Australia, English language historical and philological research gives English speaking students and practitioners our best access to CM's textual tradition. This kind of research, however, rarely assists CM clinicians with issues of medical practice. The model for medical history research separated scholarship from practice in the nineteenth century, so the historical and textual research of China's medical traditions normally avoids discussing the implications of medical theorising for therapeutic interventions. Nevertheless, it can show how current concepts and practices developed from CM's pre-modern literature; how CM's early texts reveal a unique image of the body; and how the Chinese medical body image gives internal intelligibility to the discipline's conceptual models and therapeutic methods.

In an era where we recognise the integration of physical, psychological and social factors in health management, CM's representations of the medical body have explanatory insight and therapeutic potential for contemporary clinicians and healthcare users. China's early notions of $q i$ (气), for example, bridge the distinction between energy and matter, and as a medical concept, $q i$ organises bodily phenomena into qualitative and directional influences and substances. $Q i$-influences and substances form the basis of the Chinese medical perspective on physical, cognitive, sensory and emotional conditions, and CM's conceptual language is linked to its therapeutic methods (Felt 2008). CM's traditional conceptions are detailed and holistic, and they explain important features of body-mind physiology, disorder and treatment.

Within this context, the paper explores some of the broad issues concerning the education and training of English speaking practitioners of CM in Australia. Those issues relate primarily to the translation of Chinese CM texts, the transmission of CM 
knowledge-practice, and the relevance of CM for contemporary Westerners. The translation of Chinese language texts in this case includes the complex cultural connotations of language, as well as the translation of classical Chinese into modern languages; the transmission of historical texts and practices from their original circumstances into twenty-first century medical practice; and the relevance of a foreign medical system and body of knowledge for the Australian healthcare context.

\section{Chinese medicine in China}

In pre-modern China there was a great diversity of health, medical and self cultivation practices and learning. Medical practice was passed down familial lines and consisted of secret remedies and herbal formulas that were prescribed symptomatically. Students of medicine undertook apprenticeships with reputable doctors and the first step for literate and scholarly medical training was to memorise the medical classics by heart. However, by the early twentieth century, many influential Chinese considered all traditional medical practices backward and superstitious relics of Imperial China (Croizier 1975 \& 1976), and with the end of the Imperial era in 1912, scientific medicine from the West began to take hold of the public mind.

At that time, CM was far from being standardised, institutionalised or scientific. It was not a single, cohesive, coherent system of medicine, but a mass of complex and disparate currents that seemed to neglect objective methods and data, and even anatomical structures and physical mechanisms. Proponents of Western bioscientific medicine almost convinced the new Chinese Republican government to eradicate it altogether, but instead CM was modernised. To achieve this, the modernisation of CM during the twentieth century instigated a number of significant changes that have affected the way we encounter CM in the West.

During China's revolutionary period in the middle of the last century, CM was transformed into a more systematic medical discipline. The Communist government instigated a wave of revisions that were ideological and designed to integrate $\mathrm{CM}$ with 'Western medicine,' a term used by the Chinese to refer to the scientific medicine that had begun to filter into China via European missionaries and merchants well before the twentieth century. The two main revisionary influences during the twentieth century were modernisation and integration; with the coming to power of the Communist 
regime in 1949, much of the impetus for modernisation came from the new government policies to integrate $\mathrm{CM}$ with Western medicine.

Recognising that the successful integration of Chinese and Western medicine required a fairly seamless and coherent approach to healthcare, the Chinese Communist Party (CCP) undertook an enormous effort to archive, research, revise and modernise CM. For that purpose the $\mathrm{CCP}$ initiated the collection of pre-modern texts; produced new editions of classic texts and reprinted some ancient collections that had been unavailable for centuries; and it made a concerted effort to record the folk and family lineage practices of the masses because it was felt that all these materials and practices were the communal property of the Chinese people (Porkert 1976; Taylor 2005).

With the CCP's sociopolitical agenda behind it, the modernisation of CM comprised a number of major changes which can be encapsulated under three broad processes: institutionalisation, standardisation, and scientisation and research (for more on these changes see Farquhar 1992; Hsu 1999; Scheid 2002b \& 2007; Taylor 2005). These had the effect of organising and systematising CM, while at the same time emphasising its 'scientific' aspects. Their application to CM helped to adjust traditional concepts and methods and to make CM more suitable for integration with Western medicine.

\section{Institutionalisation}

Today, and for the first time in China's history, CM is fully institutionalised: Chinese medical training, qualification and practice are run by the State, and Chinese medical care is mainly hospital based. CM colleges and clinics were first established on a nationwide scale in the late 1950s and early 1960s as government run work units (Hsu 2000: 207). Today, those colleges and clinics are CM Universities and CM Hospitals.

In the latter half of the twentieth century, the new centralised training curriculum needed a suite of teaching materials, and CM textbooks were created for the first time. The architects of China's twentieth century revisions sought areas of crossover between $\mathrm{CM}$ and Western medicine and these were reflected in CM's new curriculum and materials.

\section{Standardisation}

Twentieth century revisions in the People's Republic of China's (PRC) included a 
number of projects aimed at standardising $\mathrm{CM}$, starting with education and training and extending to terminologies, theoretical principles and therapeutic content. CM textbooks had to adjust their representations of Chinese medical concepts to demonstrate their connections with biomedical terms and categories, and all the new topics, theories and applications were standardised to comply with the centralised training curriculum.

The standardisation of terms and concepts adopted biomedical connotations where possible and this trend spread to their translation into English. For example, 病 bing (illness) is translated as disease; 证 zheng (pattern) as syndrome; 消渴 xiaoke (wasting and thirsting) as diabetes. Other projects have standardised the location of acupoints, the analysis of illnesses and diseases according to their 气 $q i$ patterns, and the strategies for treatments. In the twenty-first century, China's internal revisions are going global: in Beijing, the CM State Administration has developed a 'World Standard of Chinese Medicine Undergraduate Education' document to guide CM training outside China (Shan 2009).

\section{Scientisation and Research}

The general acceptance of the scientific approach today has meant that its methods and the knowledge produced by them are thought to be factual, unbiased, reliable and widely applicable. To call a medical system 'non-scientific' today is to damn it as 'arbitrary, irrational, unsystematic, misguided, ineffective and probably a danger to health' (Cunningham \& Andrews 1997: 7). Science was applied to CM to eradicate content deemed to be superstitious and out-dated, to correct pre-modern concepts and diagnostic methods, to align $\mathrm{CM}$ with bioscientific methods and $\mathrm{CM}$ terms with biomedical terms, and to evaluate the safety and efficacy of CM treatments.

Some medical scientists have argued that it is possible to use and test CM from within a bioscientific framework, while also arguing that scientific clinical research could establish the efficacy of CM therapies and help develop a raft of new pharmaceuticals. The application of bioscientific methods usually meant removing CM's traditional principles and concepts, and this was considered a win-win process. Science would make CM more efficient and more effective; and science could show the world that $\mathrm{CM}$ has a great deal to offer medical care systems everywhere. 
Considering CM's historical legacy, its complex and disparate currents, its neglect of physical structures and mechanisms, its incompatible assumptions and methodological dissonance with biomedicine, the application of bioscientific principles to CM is a persuasive option for the contemporary healthcare industry. Pragmatists argue that CM should jettison the traditional packaging and adapt its therapeutic tools and substances to the biomedical paradigm. Many Chinese medical texts nowadays are written to this end, for clinicians and researchers who want to utilise CM within a Western biomedical framework (such as Chang 1992; Chen 1994; Chen \& Chen 2004; Hou 1995; Liu \& Liu 1998; Zhang 2003).

Even before the twentieth century, China's indigenous medicine already had a long history of revisions that were designed to eradicate dogma and superstition. The PRC's programs of scientisation, standardisation and institutionalisation characterise the latest overhaul, which began about one hundred years ago. Twentieth century changes have 'modernised' CM, making it a more suitable discipline for integration with Western biomedicine.

\section{Chinese medicine in Australia}

In the mid-1800's Chinese gold miners began to settle in Australia. By 1887, 'there were fifty Chinese herbal medicine practitioners on the Victorian goldfields, and by 1911 Chinese herbal remedies were available in Australia with English labels and directions' (Bensoussan \& Myers 1996: 22). But it was not until the early 1970's that Chinese medicine began to attract mainstream interest, after the opening of Australia's diplomatic relations with China and the beginnings of acupuncture training in Sydney. Late in the 1980s, a Sydney acupuncture college developed its four-year part-time course into an undergraduate degree program for accreditation by the NSW Higher Education Board. From 1992 that program, followed by others incorporating Chinese herbal medicine was absorbed into the Science and Health Sciences Faculties of four universities in Sydney and Melbourne.

Some of the PRC's first training program textbooks became the precursors of some of the first English language textbooks available in Australia and the West. For example, Manfred Porkert's The Theoretical Foundations of Chinese Medicine (1974) was based on the Outline of TCM and Compendium of TCM (Nanjing Academy of TCM 1958; Nanjing College of TCM 1959); John O’Connor and Dan Bensky's Acupuncture: A 
Comprehensive Text (Shanghai College of TCM 1981) was a translation of the Textbook of Acu-Moxi-Therapy (Shanghai College of Traditional Medicine, Acu-Moxi-Therapy Teaching Unit 1962 \& 1974); and Nathan Sivin's Traditional Medicine in Contemporary China (1987) included a discussion and partial translation of The Revised Outline of TCM (Beijing College of TCM 1972). During the 1970s and 1980s these three books were the only PRC-based CM texts available in English. They introduced Australians and other Westerners to the twentieth century's revised CM, known outside China as 'Traditional Chinese Medicine' (TCM). Today, TCM is the Chinese medical orthodoxy in China and the West.

From its modest beginnings in Australia in the 1970's the training of CM clinicians has moved from privately owned colleges into the university system (1992) and the profession is moving towards national registration in 2012. Australia's much smaller scale institutionalisation of $\mathrm{CM}$ has nevertheless led to some significant gains for the discipline and the profession, such as more access to university resources that can provide greater opportunities for teaching and research. The tertiary education and training of practitioners in Australia has also improved CM's public profile, aligned it more closely with 'mainstream' healthcare, and when combined with national registration, public safety and confidence in $\mathrm{CM}$ will be enhanced.

Nevertheless, in the Australian context CM is still only one tiny fish in a very large tertiary education and health services pond; and, unlike China's integrated medical degree model, which trains Chinese students in both CM and biomedicine, Australia's $\mathrm{CM}$ degrees qualify their graduates to practice CM only. Moreover, China's medical graduates study and practice within a health system that supports both CM and biomedicine. In Australia there are no universities and hospitals solely devoted to CM; within universities $\mathrm{CM}$ remains too small to merit school or faculty status; and there are also no CM facilities within Australia's government run hospital system. Thus, while in China CM has an established academic profile, a large base of reference and research, and the support of government policy and instruments, in Australia CM's move into the university setting has by default, aligned it with bioscientific health and medical programs and their research agendas.

Standardised concepts, terms, translations, acupoint locations, diagnostic parameters and therapeutic strategies have made some significant inroads into English language 
education materials. For a discipline whose discourses and methods have been developing over many centuries, standardisation offers a number of benefits. It gives $\mathrm{CM}$ a firm foundation for education and clinical learning; and it improves communication for education and medical practice, and between Chinese and English speaking countries. Standardisation often means biomedicalising CM's content and categories, which allows clinicians and researchers to apply $\mathrm{CM}$ therapies to biomedical diagnoses. Thus, from a biomedical perspective, standardisation should improve the inter-examiner reliability of CM practice and research.

However, the biomedical re-interpretation of traditional terms that has taken place through the standardisation of terminology has lead to a sense that CM is essentially similar to biomedicine (Waldram 2000). When guided by bioscientific disease classification, the standardisation of CM terms displaces polysemous terms with more fixed, biomedical meanings and relations. It removes their original contexts and meanings and decouples them from the Chinese medical archive- their conceptual histories and contexts. Biomedical standardisation thereby erases thousands of years of diversity, and in doing so it removes the tradition's inbuilt flexibility (Farquhar 1987).

The precision of biomedical technologies and research methods promise objective, factual information, and they offer a systematic way of investigating complex systems by isolating and testing its more simple parts or factors. Evidence based medicine relies on these methods and technologies and overrides all other criteria for therapeutic safety, efficacy and best practice. Because scientific clinical research determines therapeutic safety and efficacy, it also determines ethical medical practice.

The investigation of CM using bioscientific research methods, however, has proven to be problematic in a number of ways. The research applied to $\mathrm{CM}$ often consists of unpacking a clinical event, which is itself a collection of complex processes, to systematically test an isolated factor. For example, measuring the effects on a biomedical disease entity of a single acu-point, or a fixed protocol, or a single active constituent derived from one of CM's medicinal substances. CM's practice methods and therapies are largely incompatible with this kind of research. Classic herbal formulas are complex interventions that are structured to address patterns of illness and dysfunction; and both acupuncture and herbal prescriptions are adjusted to individual presentations that change from one clinical appointment to the next. 
Furthermore, $\mathrm{CM}$ diagnosis does not rely on quantitative data derived from measuring technologies and other objective methods. In fact, ordinary sensory information - the patient's subjective experiences and perceptions, and the clinician's own observations and interpretations - were thought to be sufficient to understand the nature of illness and discern the mechanisms of dysfunction. The methodological constraints required by scientific research ignore CM's diagnostic reasoning and basic principles of practice, alter traditional methods and standardise treatment protocols (to reduce variables for example), and remove CM's flexibility and responsiveness to clinical changes and variations (Bian \& Moher 2008).

\section{The options for Chinese medicine in Australia}

$\mathrm{CM}$ in Australia does not have the depth and maturity of CM in China. In Australia we have only a few decades of marginalised practice, a very small senior practitioner population, limited access to pre-modern texts, and a relatively slight hold on the public mind. Our ability to study and practice CM in Australia is affected by the transmission of CM from its traditional contexts. Specifically, these factors are related to CM's language and literature, its history and development, its philosophical and methodological assumptions, and its viability in the contemporary sociopolitical medical setting.

To address the problems of language and translation, English speaking students of CM could learn and translate Chinese. However, China's early medical texts are notoriously compact and difficult, even for native Chinese speakers. Consequently, an enormous number of editions, revisions, commentaries and interpretations have accumulated around them over the centuries, and the few English language translations we have of these materials are of variable quality (Sivin 1993: 207).

Westerners who wish to practice traditional CM strive to gain an understanding of the discipline that corresponds to its established therapeutic methods. Traditionalism and idealism aside, there are some pragmatic reasons to study traditional discourses. Our ability to recognise and understand the traditional Chinese medical body and its representations in the pre-modern literature fundamentally changes our clinical encounters with our patients, and has ramifications for diagnostic and therapeutic decision making (Farquhar 1994; Scheid 2002a \& 2006; Zhou \& Zhang 2005). By 
studying Chinese medical history and its pre-modern texts we expand our understanding of how health, disease and the medical body can be conceptualised: this connects us with the tradition and allows us to incorporate a raft of time-honoured clinical methods and techniques.

However, the odds against contemporary English speaking practitioners understanding the Chinese medical tradition are high. To facilitate the educational and clinical transmission of CM today China has 'scientised' its textbooks, and many of the historical and cultural contexts and meanings of their content have been removed. Similarly, CM textbooks in English have changed traditional concepts: the biomedicalisation of pre-modern terms and concepts dislocates them from CM's established therapeutic methods and disrupts the discipline's internal intelligibility. The changes and issues described above present a significant challenge for the transmission of $\mathrm{CM}$ and its preservation as a distinct medical system in contemporary English speaking countries such as Australia.

While it is difficult to predict the course of CM's global emergence in any detail, commentators such as Volker Scheid (1999) postulate three possible scenarios. I mention them since they broadly apply to the Australian context where all three to some extent are underway. The first would see CM institutionalised. Although it is unlikely Australia will follow China's model of integration and institutionalisation, Australian universities now have CM degree courses; the Victorian state government currently registers its practitioners, and national registration will be in place in Australia in 2012.

A second possible scenario would see biomedicine assimilate CM. Biomedicalpharmaceutical researchers would selectively 'discover' the active constituents in Chinese medicinal substances and employ Chinese medical techniques wherever they might benefit health outcomes. Assimilation would replace traditional diagnostic reasoning and methods with bioscientific ones, and effectively dismantle $\mathrm{CM}$ as a distinct form of medical practice. Scheid's third scenario sees CM ignoring mainstream political and economic power, and continuing its traditions 'for the sake of clinical efficacy’ (1999: SIV10). With little knowledge of its historical trends and developments CM practitioners today must either reinvent the wheel or replace it with dissonant constructs. Conversely, with a firmer grasp of the Chinese medical tradition the profession would be in a better position to negotiate what is learned, taught, practiced, 
and researched.

\section{Conclusions}

Today, Western biomedicine is the dominant medical discourse in healthcare systems worldwide. In China and in the rest of the world, CM has been revising its content and concepts to adopt a more mainstream, bioscientific perspective. These revisions make CM more suitable for integration or assimilation with biomedicine, and for investigation using scientific perspectives and methods. The revisions that took place over the last century are ongoing - they have aligned $\mathrm{CM}$ with a more biomedical perspective, they have had important consequences for the transmission of CM in Australia and the West, and are changing $\mathrm{CM}$ worldwide.

Twentieth century changes to CM have organised and systematised many of the disparate medical currents that developed in pre-modern China. Over the last hundred years CM's overhaul within China was driven by the sociopolitical imperatives to modernise and integrate its healthcare system. This push to modernise CM is not inconsistent with previous state instigated revisions that occurred periodically over its long history and that were designed to eradicate dogma and superstition. In Australia, $\mathrm{CM}$ as a distinct medical discipline does not have a strong cultural basis or presence, and CM training is only a few decades old. Thus, in contemporary Western settings, $\mathrm{CM}$ must prove itself to a sceptical biomedical health industry while at the same time promoting its complementary approach to the practice of medicine (Chi 1994).

The education and practice of $\mathrm{CM}$ and biomedicine are likely to co-exist independently in Australia for quite some time, and the question for the future of CM in Australia is how best to professionalise the discipline and negotiate our way. The path of least resistance politically and educationally is to biomedicalise CM. However, even though biomedicalisation offers some sociopolitical kudos and some practical educational shortcuts, it has also lead to unworkable simplifications and methodological failures. Instead, building access to the tradition's primary sources can reveal internal principles and intelligibility that support its methods of practice and continue the evolution of the field and its traditions. Allied with biomedicine, the distinctive features and methods of traditional CM may well provide real benefits for the Australian healthcare system, users and costs. 


\section{Reference List}

Beijing College of TCM 1972, Revised Outline of Chinese Medicine, Beijing College of TCM, Beijing (in Chinese).

Bensoussan, A. \& Myers, S. P. 1996, Towards a Safer Choice: The Practice of Traditional Chinese Medicine In Australia, University of Western Sydney, Macarthur, Sydney.

Bian, Z. \& Moher, D. 2008, 'Clinical Studies and Randomized Controlled Trials in Chinese Herbal Medicine: A Historical and Contemporary Review - Part Two,' Chinese Medicine Times eJournal, vol. 3, no. 3. Online, available: http://www.chinesemedicinetimes.com/ [accessed 1 September 2008].

Chang, M. Y. 1992, Anticancer Medicinal Herbs, trans. Y. Bai. Hunan Science and Technology Press, Hunan, China.

Chen, J. D. 1994, Treatment of Diabetes with Traditional Chinese Medicine, trans. Y. K. Sun, S. H. Zhou \& Y. B. Lu. Shandong Science and Technology Press, Jinan, China.

Chen, J. K. \& Chen, T. T. 2004, Chinese Medical Herbology and Pharmacology. Art of Medicine Press, City of Industry, California.

Chi, C. H. 1994, 'Integrating Traditional Medicine into Modern Health Care Systems: Examining the Role of Chinese Medicine in Taiwan,' Social Science and Medicine, vol. 39, no. 3: 307-332.

Croizier, R. C. 1975, 'Medicine and Modernization in China: An Historical Overview,' in Medicine In Chinese Cultures: Comparative Studies of Health Care in Chinese and Other Societies. Papers and Discussions from a Conference Held in Seattle, Washington, USA, February 1974, (eds) A. Kleinman, P. Kunstadter, E. R. Alexander \& J .L. Gale. U.S. Dept. of Health, Education, and Welfare, Public Health Service, National Institute of Health, Washington: 21-35.

Croizier, R. C. 1976, 'The Ideology of Medical Revivalism in Modern China,' in Asian Medical Systems: A Comparative Study, (ed.) C. Leslie. University of California Press, Berkeley: 341-355.

Cunningham, A. \& Andrews, B. 1997, 'Western Medicine as Contested Knowledge,' in Western Medicine as Contested Knowledge, (eds) A. Cunningham \& B. Andrews. Manchester University Press, Manchester.

Farquhar, J. 1987, 'Problems of Knowledge in Contemporary Chinese Medical Discourse,' Social Science and Medicine, vol. 24, no. 12: 1013-1021.

Farquhar, J. 1992, 'Time and Text: Approaching Chinese Medical Practice Through Analysis of a Published Case,' in Paths to Asian Medical Knowledge, (eds) L. Young \& A. Young. University of California Press, Berkeley \& Los Angeles: 62-73.

Farquhar, J. 1994, Knowing Practice: The Clinical Encounter of Chinese Medicine. Westview Press, Boulder.

Felt, R. 2008, 'Is Qi Energy?,' in Theime Almanac 2008: Acupuncture and Chinese Medicine, (eds) M. McCarthy \& S. Birch. Thieme, Stuttgart: 304-308.

Hou, J. L. 1995, Treatments of Gastrorintestranal Diseases in Traditional Chinese Medicine. Academy Press, Beijing.

Hsu, E. 1999, The Transmission of Chinese Medicine. Cambridge University Press, Cambridge.

Hsu, E. 2000, 'Spirit (Shen), Styles of Knowing, and Authority in Contemporary Chinese Medicine,' Culture, Medicine and Psychiatry, vol. 24, no. 2: 197-229.

Liu, Y. C. \& Liu, Z. W. 1998, Basic Theories of Traditional Chinese Medicine, Academy Press, Beijing. Nanjing Academy of TCM 1958, Outline of TCM. People's Medical Publishing, Beijing (in Chinese).

Nanjing College of TCM 1959, Compendium of TCM. People's Hygiene Press, Beijing (in Chinese).

Porkert, M. 1974, The Theoretical Foundations of Chinese Medicine: Systems of Correspondence. First ed. MIT Press, Cambridge, MA.

Porkert, M. 1976, 'The Intellectual and Social Impulses Behind the Evolution of Traditional Chinese Medicine,' in Asian Medical Systems: A Comparative Study, (ed.) C. Leslie. University of California Press, Berkeley: 63-76.

Scheid, V. 1999, 'The Globalisation of Chinese Medicine,' The Lancet, vol. 354, supplement 4, December: SIV10-SIV10.

2002a, Chinese Medicine in Contemporary China: Plurality and Synthesis. Duke University Press, Durham, NC, \& London. 2002b, 'Remodeling the Arsenal of Chinese Medicine: Shared Pasts, Alternative Futures,' Annals, American Academy of Political and Social Science, vol. 583, September: 136-159. 2006, 'Chinese Medicine and the Problem of Tradition,' Asian Medicine, Tradition and Modernity, vol. 2 , no. 1: 59-71. 2007, Currents of Tradition in Chinese Medicine 1626-2006. Eastland Press, Seattle. 
Shan, J. 2009, 'New TCM Standard Created,' China Daily: The National English Language Newspaper, October 13: 1. Online, available: http://www.chinadaily.com.cn/ [accessed 1 November 2009].

Shanghai College of TCM 1981, Acupuncture: A Comprehensive Text, trans. J. O’Connor \& D. Bensky. Eastland Press, Chicago.

Shanghai College of Traditional Medicine (Acu-Moxi-Therapy Teaching Unit) (1962 \& 1974), Textbook of Acu-Moxi-Therapy. Shanghai Science and Technology Press, Shanghai (in Chinese).

Sivin, N. 1987, Traditional Medicine in Contemporary China: a Partial Translation of Revised Outline of Chinese Medicine (1972): With an Introductory Study on Change in Present Day and Early Medicine. Center for Chinese Studies, University of Michigan, Ann Arbor. 1993, 'Huang Ti Nei Ching,' in Early Chinese Texts: A Bibliographical Guide, (ed.) M. Loewe. Society for the Study of Early China; Institute of East Asian Studies, University of California, Berkeley: 196-215.

Taylor, K. 2005, Chinese Medicine in Early Communist China, 1945-63: A Medicine of Revolution. RoutledgeCurzon, London.

Unschuld, P. U. 1993, 'History of Chinese Medicine,' in The Cambridge World History of Human Disease, (ed.) K. F. Kiple. Cambridge University Press, Cambridge: 20-27.

Waldram, J. B. 2000, 'The Efficacy of Traditional Medicine: Current Theoretical and Methodological Issues,' Medical Anthropology Quarterly, vol. 14, no. 4: 603-625.

Zhang, D. Z. 2003, Treating Toxico-Side Effects of Radiotherapy and Chemotherapy with Integrative Traditional Chinese and Western Medicine, trans. W. Lu \& P. Lin. People's Medical Publishing House, Beijing.

Zhou, F. W. \& Zhang, Q. W. 2005, 'The Path of the Old Chinese Doctors,' The Lantern, vol. 2, no. 2: 613. 


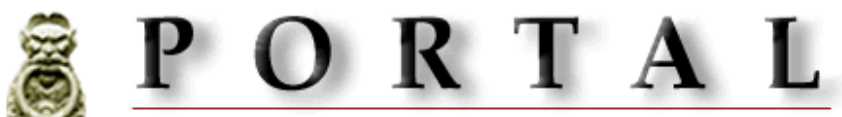

Journal of Multidisciplinary International Studies

Home About Log In Register Search Current Archives

Announcements

Home $>$ Vol 8, No 3 (2011)

\section{PORTAL Journal of Multidisciplinary International} Studies

Portal Journal of Multidisciplinary International Studies is a fully peer-reviewed journal dedicated to the publishing of scholarly articles from practitioners ofand dissenters from-international, regional, area, migration and ethnic studies, and it is also dedicated to providing a space for the work of cultural producers interested in the internationalization of cultures. International Studies in Portal refers to studies of contemporary societies and cultures in relation to processes of transnationalization, polyculturation, transculturation, transmigration, globalization, and anti-globalization, and to material and imaginative responses by people and communities to those processes. Portal aims to achieve a genuinely multidisciplinary mix of approaches to international studies by scholars working in the Humanities, Social Sciences, and Cultural Studies, and by cultural producers working anywhere. The journal's commitment to fashioning a genuinely "international" studies venue is also reflected in our willingness to publish critical and creative work in English as well as in Chinese, French, German, Indonesian, Italian, Japanese, and Spanish. Portal is categorized as an A journal in the Australian Research Council's Excellence in Research for Australia [ERA] rankings of peerreviewed journals.

Vol 8, No 3 (2011): Global Climate Change Policy: PostCopenhagen Discord

Table of Contents

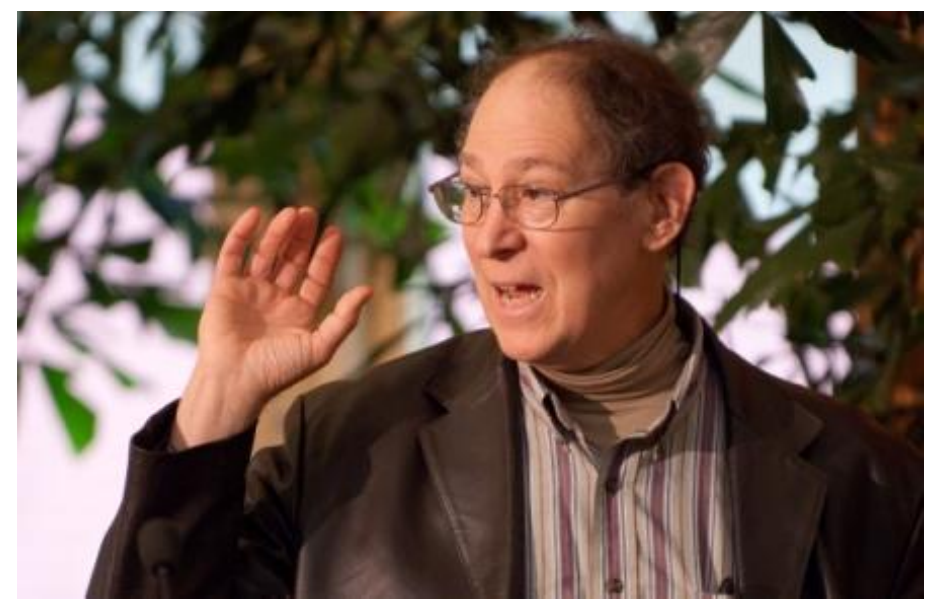

Special Issue guest edited by Chris Riedy and lan M. Mcgregor.

The late Stephen Schneider (c) Linda A. Cicero / Stanford News Service.

\section{$\mathrm{e}^{\text {Press }}$}

Search

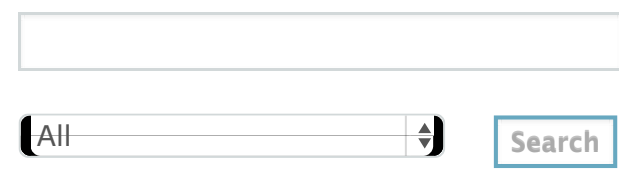

Browse

- By Issue

- By Author

- By Title

- Other Journals

Information

- For Readers

- For Authors

- For Librarians

Journal Help

User

Username

Password

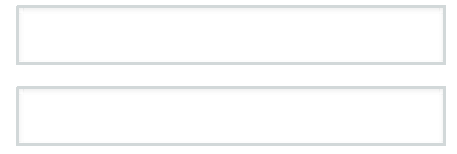

Log In $\square$ Remember me

Notifications

- View

- Subscribe / Unsubscribe

Keywords

Australia China climate Change Europe Exile

Gender Hyperworld(s) Indonesia Japan

Knowledge Language Literature Memory

Migration Poetry Politics Translation exile gender memory women 